\title{
Machine learning and materials discovery
}

Gus Hart

The relative accuracy and speed of density functional calculations have transformed computational materials science and enabled the creation of large databases of computed materials properties. But true "materials by design" or in- silico materials discovery has not yet been realized, though there are isolated success stories. It seems likely that into make computational discovery of new materials possible, or to discover materials engineering routes to improve already- deployed materials, a brute force approach will not be practical — some other paradigm will be required. Machine learning, so successful in some other application areas, is an intriguing and promising idea, but there are hurdles to overcome. There are two important differences between the standard machine learning problems of image recognition, voice recognition, etc., and materials prediction. In the first instance, we cannot afford the typical accuracy tradeoff - materials predictions are not useful without meeting a high accuracy target; the energy difference of competing phases is often very small, requiring high fidelity in the models. The second difference is the amount of training data - we don't have "big data."e How do we move forward? In this talk I will review the state of the art in this emerging discipline and show some results from BYU's Materials Simulation Group efforts in this area.

*This work is supported by ONR (MURI N00014-13-1-0635). 